\title{
An Improved Method of Numerical Simulation of Resin Transfer Molding for Resin-Based Composite Materials Based on Unstructured Grid
}

\author{
Fei Shi ${ }^{1}$, Zhong Zhao ${ }^{1}$ and Xianghuai Dong ${ }^{2}$ \\ ${ }^{1}$ NingBo University of Technology, Ningbo 315016, China \\ ${ }^{2}$ Shanghai Jiao Tong University, Shanghai 200030, China
}

\begin{abstract}
The authors have a lot researches about numerical simulation of resin transfer molding for resin-based composite materials based on unstructured grid in the past. In these researches the authors found while the grid is orthogonal, the simulation is agreed with facts. But the grid is nonorthogonal, the energy equation has great error due to being simplified, and the results are wrong. The mesh distortion is larger, the deviations are bigger. Therefore, this paper conducted a study about the calculation methods of the energy equation, and validated through case reasonableness and accuracy of the calculation method. The results show the improvement is effective.
\end{abstract}

Keywords-numerical simulation; resin-based composite materials; unstructured grid; control volume / finite element $\operatorname{method}(\mathrm{CV} / \mathrm{FEM})$

\section{INTRODUCTION}

When simulating resin transfer molding of Resin-based composite materials using the control volume / finite element method (CV/FEM) in order to adapt to the different shape of parts, non-structured grids are often used, but calculated precision and grids has the very big relations due to diffusion of the energy equation. When the computational grid is the structured grid, the connection between the adjacent nodes and the common interface of control volume is orthogonal. So, the cross diffusion term of the energy equation is equal to zero. When grids are unstructured, the connection between the nodes and the boundary of the control volume is non-orthogonal, then cross diffusion is not zero, especially when the crossover is far from orthogonal, the cross diffusion has a great influence on the numerical results.

In most of the previous researches [1,2,3,4,5,6], the simulations were simplified by calculating the flow under the two-dimensional(2D) setting and assuming the heat diffusion to be only in the plane. Another previous researches [7,8,9]developed 2.5-dimentional (2.5D) non-isothermal models, which include the flow in plane and heat diffusion in the thickness for the thin parts based on the CV/FEM. In some other researches [10,11], the 3D simulation of filling was performed during the RTM using the CV/FEM based on regular structured mesh. There are also previous studies, say Refs. [12,13], focusing on applying the 3D Galerkin finite element methods to simulate the filling stage or the curing stage. Ref. [14] thinks that control volume method is no way to directly calculate the cross diffusion of common interface.
Therefore, the cross diffusion term is not directly calculated from the integral of the diffusion term on the common interface.

The author has done a lot of research on the numerical simulation of Resin-based composite materials [15-18]. In these studies, the numerical simulation can help the researches know and understand the RTM process. These studies contribute to find the relevant parameters affecting the process and optimize the way. In these researches, in order to improve the calculation accuracy, the structured hexahedral mesh was used and hence the flexibility of dealing with complicated shapes was not sufficient. Therefore.

\section{CONTROL EQUATIONS}

In this section, we present the basic control equations. For the non-isothermal RTM process under investigation, we make the following assumptions:

(1) The resin is an uncompressed liquid and its viscosity is a function of the temperature and curing ratio.

(2) The temperature of the resin and fiber is identical at each position.

(3) There is no resin before the flow front and the control volume is fully saturated after the flow front.

(4) There is a quasi-steady time window, in which the viscosity dissipation energy can be neglected.

(5) Resin stops flow at the curing stage.

\section{A. The Control Equation}

1) The flow equation

At filling stage, Darcy's equation, which describes how the resin flows in the fiber, is still applicable. In the case, Darcy's law, which describes the flow within the mold, can be written as follows:

$$
\vec{v}=-\frac{1}{\mu}[K] \nabla P
$$

Where, $\vec{v}$ is the Darcy velocity vector, $\mathrm{K}$ is the permeability tensor, $\nabla P$ is pressure gradient, which is a 
function of the resin temperature and degree of cure given below:

$$
\mu(T, \alpha)=\mu_{0} \mathrm{e}^{\frac{E_{\mu}}{R T}}\left(\frac{\alpha_{g}}{\alpha_{g}-\alpha}\right)^{d_{1}+d_{2} \alpha}
$$

Where, $\mu_{0}$ is the initial degree of viscosity, $E_{\mu}$ is the activation energy of resin, $\alpha_{g}$ is the degree of cure of the resin gel point, $d_{1}$ and $d_{2}$ are two constants, $\mathrm{R}$ is the gas constant, and $\mathrm{T}$ and $\mathrm{a}$ are the temperature and the degree of cure, respectively.

Based on the quasi-steady state assumption, the mass balance equation at each node is given by:

$$
\phi \frac{\partial \rho}{\partial t}=-\nabla \cdot \vec{v}
$$

Where, $\rho$ is the mass density, $\mathrm{t}$ is time.

The possible boundary conditions are:

(a) $p=p_{0}$ for constant pressure injection or $u=u_{0}$ for constant velocity injection at the inlet;

(b) $p=0$ at the flow front;

(c) $\partial p / \partial n=0$ at the mold wall

2) The energy equation

At the same time, it is supposed that the heat convection of the resin and fiber occurs and the temperature is equal at resin and fiber everywhere. So the energy equation is written as :

$$
\begin{aligned}
& {\left[\phi \rho_{\mathrm{r}} c_{\mathrm{\rho r}}+(1-\phi) \rho_{\mathrm{f}} c_{\mathrm{\rho f}}\right] \frac{\partial T}{\partial t}+\rho_{\mathrm{r}} c_{\mathrm{\rho r}}\left(u \frac{\partial T}{\partial x}+v \frac{\partial T}{\partial y}+w \frac{\partial T}{\partial z}\right)} \\
& =k_{\mathrm{Lx}} \frac{\partial^{2} T}{\partial x^{2}}+k_{\mathrm{Ly}} \frac{\partial^{2} T}{\partial y^{2}}+k_{\mathrm{L} z} \frac{\partial^{2} T}{\partial z^{2}}+\phi \dot{s}
\end{aligned}
$$

Where, $\phi$ is the porosity, $\rho_{\mathrm{r}}$ and $\rho_{\mathrm{f}}$ are the resin density and the fiber density, respectively, $c_{\mathrm{pr}}$ and $c_{\mathrm{pf}}$ are the resin specific heat and the fiber specific heat, respectively, $u, v, w$ is the velocity in $\mathrm{x}, \mathrm{y}, \mathrm{z}$ directions, $k_{\mathrm{Lx}}, k_{\mathrm{Ly}}$, $k_{\mathrm{Lz}}$ is the whole heat conductivity of fiber and resin, which can be expressed as:

$$
k_{\mathrm{Lx}}=\frac{k_{\mathrm{rx}} k_{\mathrm{fx}}}{k_{\mathrm{rx}} W_{\mathrm{f}}+k_{\mathrm{fx}} W_{\mathrm{r}}}
$$

$$
W_{\mathrm{r}}=\frac{\frac{\phi}{\rho_{\mathrm{f}}}}{\frac{\phi}{\rho_{\mathrm{f}}}+\frac{1-\phi}{\rho_{\mathrm{r}}}}, \quad W_{\mathrm{f}}=1-W_{\mathrm{r}}
$$

The boundary conditions are given as:

(1) $T=T_{\mathrm{r} 0}$ at the inlet gate

(2) $\left.k_{\mathrm{L}} \frac{\partial T}{\partial n}\right|_{\mathrm{rr}}=(1-\phi) \rho_{\mathrm{r}} c_{\mathrm{pr}} \overrightarrow{u-}\left(T_{\mathrm{r}}-T\right)$ at the flow front;

(3) $T=T_{\mathrm{m}}$ at the mold wall.

3) The chemical equation

While resin is curing, the resin chemical reaction follows the chemical equation and that is written as :

$$
\phi \frac{\partial \alpha}{\partial t}+u \frac{\partial \alpha}{\partial x}+v \frac{\partial \alpha}{\partial y}+w \frac{\partial \alpha}{\partial z}=\phi f\left(\alpha, T_{\mathrm{r}}\right)
$$

The boundary conditions are given by

(a) $\alpha=\alpha_{0}$ at the inlet;

(b) $\frac{d}{d t}(\omega \alpha)=\omega f\left(\alpha, T_{\mathrm{r}}\right)$ at the flow front,

\section{B. The Numerical Formulation}

Before the control equations are used to simulate the RTM process, it must be integrated in the control volume. At the past, the structure mesh is good for part of rule shape. The calculation accuracy is ensured, also. While the part is not rule, the structure mesh is unfit for simulating. So, the unstructured mesh has wide adaptability for shapes of part. While the calculation accuracy is closed related with the grid. The unstructured mesh can lead to the error of calculation results. The main reason is the cross diffusion term of energy equation exists. The size of the value has closely related with the orthogonal relation between the element nodes and the cell boundary. If the orthogonal is existence, the cross diffusion needs not considered. But it can not be ignored for the non-orthogonal grid. Especially when the cross is far away from the orthogonal, the cross diffusion has a great influence on the numerical results, which can not be zero.

Literature [14] shows that the control volume method is not a direct and accurate calculation of the cross diffusion on the common interface. Therefore, this paper does not directly calculate the cross diffusion term in the public interface from the integral of the diffusion term.

The whole derivation process is based on the orthogonal grid. By introducing the non orthogonal quantity, the improved discrete scheme is derived. 
As shown in Figure 1, the P control volume (CV) and the adjacent $\mathrm{E} \mathrm{CV}$ public interface has two, namely: FN and $\mathrm{NM}$ edge.

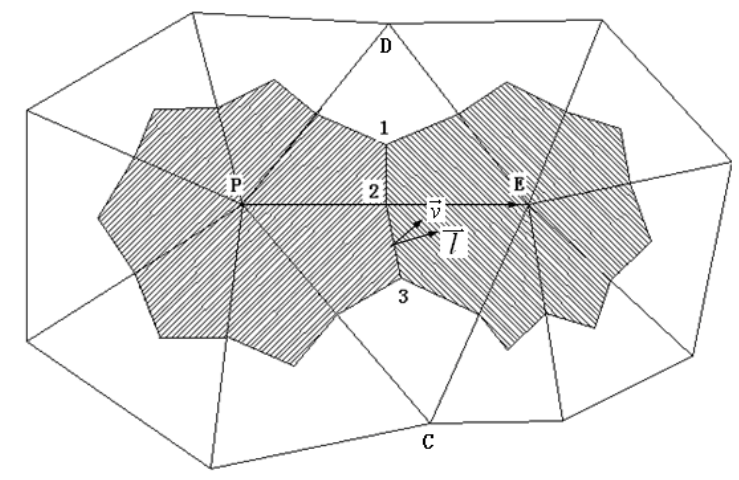

FIGURE I. THE P CV AND THE ADJACENT E CV

For the discretization of the energy equation, each part separately carries on the numerical integration, and finally all are carried on the combination assembly to obtain the final control equation the discrete format.

\section{1) Discrete of instantaneous term}

The first item on the left of the differential equation. (3) is the integral of the instantaneous term by use differential method:

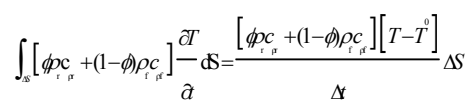

Where, 0 is the result of the calculation of the previous time step, and $\Delta S$ is the area of the control section.

\section{2) Integral of convection term}

The convection term of the energy equation is integrated by the first order upwind scheme. As Fig 1 shown:

$$
\begin{aligned}
& \int_{\Delta S} \rho_{r} c_{p r}\left(u \frac{\partial T}{\partial x}+v \frac{\partial T}{\partial y}\right) \mathrm{d} S=\int_{\Delta s} \rho_{r} c_{p r} T(\vec{v} \cdot \vec{l}) \mathrm{d} l \\
& =\rho_{\mathrm{r}} c_{\mathrm{pr}} T\left(l_{12} \overrightarrow{v_{12}} \cdot \overrightarrow{l_{12}}+l_{23} \overrightarrow{v_{23}} \cdot \overrightarrow{l_{23}}\right)
\end{aligned}
$$

Where, $\vec{l}$ is the vector of the length of the outer normal unit of the control volume boundary, $\vec{v}$ is Darcy velocity vector of the resin that is expressed on the boundary.

So:

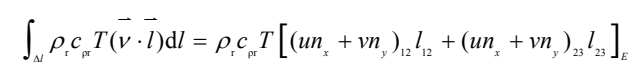

Where $u$ is the component of Darcy velocity vector in the $\mathrm{X}$ direction, and $v$ is $\mathrm{s}$ the component of Darcy velocity vector in the y direction.

For all adjacent control volume of the control volume $\mathrm{P}$, the integral of convection term in control volume can be obtained:

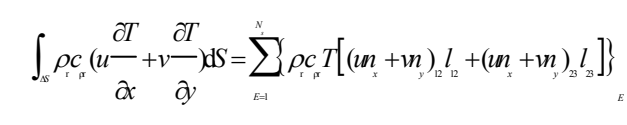

Where, $N_{S}$ is the number of controls that are adjacent to the control volume.

Also,

$$
\left\{\begin{array}{l}
u=-\frac{k_{\mathrm{x}}}{2 A \bar{\mu}} \sum_{i=1}^{3}\left(b_{i} p_{i}\right) \\
v=-\frac{k_{\mathrm{y}}}{2 A \bar{\mu}} \sum_{i=1}^{3}\left(c_{i} p_{i}\right)
\end{array}\right.
$$

Formula (9) is introduced to formula (8), and gets:

$$
\begin{aligned}
& \int_{\Delta S} \rho_{\mathrm{r}} c_{\mathrm{pr}}\left(u \frac{\partial T}{\partial x}+v \frac{\partial T}{\partial y}\right) \mathrm{d} S \\
& =\rho_{\mathrm{r}} c_{\mathrm{pr}} T \sum_{E=1}^{N}\left\{-\frac{1}{2 A_{\mathrm{PDE}} \bar{\mu}}\left(k_{\mathrm{x}} \sum_{i=1}^{3}\left(b_{i} p_{i}\right) n_{x}+k_{\mathrm{y}} \sum_{i=1}^{3}\left(c_{i} p_{i}\right) n_{y}\right)_{12} l_{12}\right. \\
& \left.-\frac{1}{2 A_{\mathrm{PCE}} \mu}\left(k_{\mathrm{x}} \sum_{i=1}^{3}\left(b_{i} p_{i}\right) n_{x}+k_{\mathrm{y}} \sum_{i=1}^{3}\left(c_{i} p_{i}\right) n_{y}\right)_{23} l_{23}\right\}_{E}
\end{aligned}
$$

3) Integration of source terms

$$
s_{T}=\phi \dot{s}=\phi \Delta H f\left(\alpha, T_{r}\right)
$$

By its linearization, it can be re written:

$$
s_{T}=s_{c}+s_{p} T
$$

Where, $s_{c}$ and $s_{p}$ Are constant, and $s_{p} \leq 0$.

Then the source terms are integral, and get:

$$
\begin{aligned}
\int_{\Delta V} \phi \dot{s} \mathrm{~d} V & =\int_{\Delta V} s_{T} \mathrm{~d} V \\
& =\int_{\Delta V}\left(s_{c}+s_{p} T\right) \mathrm{d} V \\
& =s_{c} \Delta V+s_{p} T \Delta V
\end{aligned}
$$

4) Integration of diffusion terms

The first item on the left side of the equation is heat transfer by diffusion. According to Green Equation,,

$$
\int_{\Delta S}\left(k_{\mathrm{Lx}} \frac{\partial^{2} T}{\partial x^{2}}+k_{\mathrm{Ly}} \frac{\partial^{2} T}{\partial y^{2}}\right) \mathrm{d} S=\int_{\Delta l} k_{\mathrm{Lx} \cdot}\left(\frac{\partial T}{\partial x_{i}} \cdot \vec{l}\right) \mathrm{d} l
$$

As Fig 1 shown: 


$$
\begin{aligned}
& \int_{w} k_{\mathrm{Lt}}\left(\frac{\partial T}{\partial x_{t}} \cdot \vec{l}\right) \mathrm{d} l \\
& =\left[\left(k_{\mathrm{Ls}} \frac{\partial T}{\partial x} n_{x}+k_{\mathrm{Ly}} \frac{\partial T}{\partial y} n_{y}\right)_{12} l_{12}+\left(k_{\mathrm{Lx}} \frac{\partial T}{\partial x} n_{x}+k_{\mathrm{Ly}} \frac{\partial T}{\partial y} n_{y}\right)_{2 y} l_{z 3}\right]_{\varepsilon}
\end{aligned}
$$

a) When the connection between the nodes and the boundary of the control volume is orthogonal:

$$
\left\{\begin{array}{l}
\frac{\partial T}{\partial x}=\frac{T_{E}-T_{P}}{|\overrightarrow{P E}|} n_{x} \\
\frac{\partial T}{\partial y}=\frac{T_{E}-T_{P}}{|\overrightarrow{P E}|} n_{y}
\end{array}\right.
$$

So:

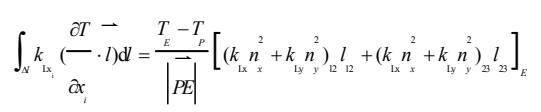

b) When the connection between the nodes and the boundary of the control volume is non-orthogonal:

$$
\begin{aligned}
& \int_{\Delta M} k_{\mathrm{Lx}_{\mathrm{i}}}\left(\frac{\partial T}{\partial x_{i}} \cdot \vec{l}\right) \mathrm{d} l \\
& =\frac{T_{E}-T_{P}}{|\overrightarrow{P E}|}\left[\left(k_{\mathrm{Lx}} n_{x}^{2}+k_{\mathrm{Ly}} n_{y}^{2}\right)_{12} l_{\mathrm{L} 2}+\left(k_{\mathrm{Lx}} n_{x}^{2}+k_{\mathrm{Ly}} n_{y}^{2}\right)_{23} l_{23}\right]_{E}+C_{\mathrm{d} \text { dir }}
\end{aligned}
$$

Where, $C_{\text {diff }}$ is cross diffusion term on a common interface.

By the formula (18), we can see that the diffusion term is mainly composed of two parts, namely, the main part and the cross diffusion $C_{\text {diff }}$ on the common interface. The main part of the diffusion is direction ratio to temperature difference between adjacent control volumes, and inclined to the distance between the two controls. Therefore, the derivation of the cross diffusion term at the common interface can also be considered.

For the cross diffusion for 23-edge of a common interface, the cross diffusion term is proportional to the temperature gradient between the two adjacent control bodies. So:

$$
C_{\text {diff, }} \propto \frac{\left(T_{\mathrm{E}}^{0}-T_{\mathrm{p}}^{0}\right)}{|\overrightarrow{P E}|}\left[\sum k_{\mathrm{Lx}_{\mathrm{x}}} n_{x_{\mathrm{x}}}\right]_{23} l_{23}
$$

Where, $T_{\mathrm{E}}^{0}$ is the temperature of $\mathrm{E}$ at the end of a time step, and $T_{P}^{0}$ is the temperature of $\mathrm{P}$ at the end of a time step.

Secondly, basing on the derivation of the diffusion terms in the front, The size of the cross diffusion term is related to the orthogonal relationship between the edge 23 and PE connections, and when the edge 23 and $\mathrm{PE}$ are connected with the orthogonal or close to the orthogonal, $C_{\text {diff }_{1}}=0$.Or else, the cross diffusion term is greater. So:

$$
C_{\text {difi }} \propto \frac{\overrightarrow{M N} \cdot \overrightarrow{P E}}{|\overrightarrow{M N}||\overrightarrow{P E}|}
$$

In the end, cross diffusion terms on the common interface can be expressed as follows:

$$
C_{\text {diff }}=\frac{\left(T_{\mathrm{E}}^{0}-T_{\mathrm{p}}^{0}\right)}{|\overrightarrow{P E}|^{2}}\left[\sum k_{\mathrm{L}_{e}} n_{x_{\mathrm{c}}}\right]_{\mathrm{MN}} l_{\mathrm{MN}} \frac{\overrightarrow{M N} \cdot \overrightarrow{P E}}{|\overrightarrow{M N}|}
$$

Formula (21) is write specific expressions, that is:

$$
C_{\text {diff }}=\frac{\left(T_{\mathrm{E}}^{0}-T_{\mathrm{p}}^{0}\right)}{|\overrightarrow{P E}|^{2}}\left(k_{\mathrm{L}} n_{x}+k_{\mathrm{Ly}} n_{y}\right)_{23} l_{23} \frac{\left[\left(X_{2}-X_{3}\right)\left(X_{\mathrm{E}}-X_{\mathrm{p}}\right)+\left(Y_{2}-Y_{3}\right)\left(Y_{\mathrm{E}}-Y_{\mathrm{p}}\right)\right]}{|\overrightarrow{23}|}
$$

Where, $\mathrm{X}$ and $\mathrm{Y}$ are the coordinates of each point, respectively.

For the cross diffusion of the 12-edge, can be expressed as follows:

$C_{\text {diff }}=\frac{\left(T_{\mathrm{E}}^{0}-T_{\mathrm{p}}^{0}\right)}{|\overrightarrow{P E}|^{2}}\left(k_{\mathrm{Lx}} n_{x}+k_{\mathrm{Ly}} n_{y}\right)_{\mathrm{L} 2} l_{\mathrm{L} 2} \frac{\left[\left(X_{2}-X_{1}\right)\left(X_{\mathrm{E}}-X_{\mathrm{p}}\right)+\left(Y_{1}-Y_{\mathrm{1}}\right)\left(Y_{\mathrm{E}}-Y_{\mathrm{p}}\right)\right]}{|\overrightarrow{12}|}$

Finally, the cross diffusion terms on the common interface between the $\mathrm{P}$ controller and the adjacent control volume E can be expressed as follows:

$$
\begin{aligned}
C_{\text {diff }}= & \frac{\left(T_{\mathrm{E}}^{0}-T_{\mathrm{p}}^{0}\right)}{|\overrightarrow{P E}|^{2}}\left\{\left(k_{\mathrm{Lr}} n_{x}+k_{\mathrm{Ly}} n_{y}\right)_{23} l_{23} \frac{\left[\left(X_{2}-X_{3}\right)\left(X_{\mathrm{E}}-X_{\mathrm{p}}\right)+\left(Y_{2}-Y_{3}\right)\left(Y_{\mathrm{E}}-Y_{\mathrm{p}}\right)\right]}{|\overrightarrow{23}|}\right. \\
& \left.+\left(k_{\mathrm{L} n_{x} n_{x}+k_{\mathrm{L} y} n_{y}}\right)_{\mathrm{L} 2} l_{\mathrm{L} 12} \frac{\left[\left(X_{2}-X_{1}\right)\left(X_{\mathrm{E}}-X_{\mathrm{p}}\right)+\left(Y_{2}-Y_{1}\right)\left(Y_{\mathrm{E}}-Y_{\mathrm{p}}\right)\right]}{|\overrightarrow{12}|}\right\}
\end{aligned}
$$

The formula (22) is introduced into the numerical integration of the diffusion term, that is, the formula (18), which can be improved by the numerical model of the energy equation under non isothermal conditions. According to the improved numerical model, the calculation accuracy of the temperature field is improved.

Integrated formula (5), formula (8), formula (11) and formula (16), in the time of the full implicit time integration scheme, and finally get the energy equation in the unstructured grid of two-dimensional discrete format: 


$$
m_{P} T_{P}=\sum_{E=1}^{N_{s}} m_{E} T_{E}+m_{R}
$$

Where, $m_{p}=\sum_{E=1}^{N} m_{E}+\frac{\left[\phi \rho_{\varepsilon} c_{\mathrm{pr}}+(1-\phi) \rho_{\mathrm{f}} c_{\mathrm{pt}}\right] \Delta S}{\Delta t}-s_{p} \Delta S$

$$
m_{R}=\frac{\left[\phi \rho_{\mathrm{r}} c_{\mathrm{pt}}+(1-\phi) \rho_{\mathrm{f}} c_{\mathrm{pf}}\right] \Delta S}{\Delta t}+s_{c} \Delta S
$$

Since the first-order upwind scheme is used, so:

$$
m_{E}=D_{E}+\operatorname{Max}\left(0,-F_{E}\right)
$$

Where,

$$
\begin{aligned}
& D_{E}=\left\{\frac{1}{|\overrightarrow{P E}|}\left[\left(k_{\mathrm{Lx}} n_{x}^{2}+k_{\mathrm{Ly}} n_{y}^{2}\right)_{12} l_{\mathrm{L} 12}+\left(k_{\mathrm{Lx}} n_{x}^{2}+k_{\mathrm{Ly}} n_{y}^{2}\right)_{23} l_{23}\right]\right\}_{E}+C_{\mathrm{diff}}
\end{aligned}
$$

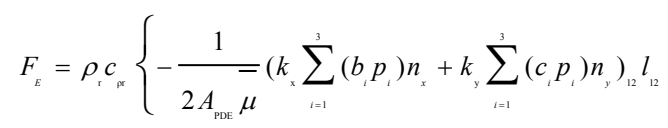

$$
\begin{aligned}
& \left.-\frac{1}{2 A_{\mathrm{PCE}} \bar{\mu}}\left(k_{\mathrm{x}} \sum_{i=1}^{3}\left(b_{i} p_{i}\right) n_{\mathrm{x}}+k_{\mathrm{y}} \sum_{i=1}^{3}\left(c_{i} p_{i}\right) n_{y}\right)_{23} l_{23}\right\}_{E}
\end{aligned}
$$

The chemical equation can be deduced like the aforementioned, and gets:

$$
n_{P} \alpha_{P}=\sum_{E=1}^{N} n_{E} \alpha_{E}+n_{R}
$$

Where, $n_{P}=\sum_{E=1}^{N} n_{E}+\frac{\phi \Delta S}{\Delta t}-f_{p} \Delta S$

$$
\begin{gathered}
n_{R}=\frac{\phi \Delta S}{\Delta t}+f_{c} \Delta S \\
n_{E}=\operatorname{Max}\left(0,-F_{E}\right) \\
F_{E}=\left\{-\frac{1}{2 A_{\mathrm{PDE}} \mu}\left[k_{\mathrm{x}} \sum_{i=1}^{3}\left(b_{i} p_{i}\right) n_{x}+k_{\mathrm{y}} \sum_{i=1}^{3}\left(c_{i} p_{i}\right) n_{y}\right]_{12} l_{\mathrm{in}}\right. \\
\left.\left.-\frac{1}{2 A_{\mathrm{PCE}} \mu} \mu_{\mathrm{x}} \sum_{i=1}^{3}\left(b_{i} p_{i}\right) n_{x}+k_{\mathrm{y}} \sum_{i=1}^{3}\left(c_{i} p_{i}\right) n_{y}\right]_{23} l_{23}\right\}_{E} \\
\text { III. CASE STUDY }
\end{gathered}
$$

The calculation flow chart is shown in Figure 2.Using the non isothermal model which was derived from the author's previous works, and the injection process and the material parameter are from literature [19], as Tab 1 shown, the numerical simulation was carried out to verify the improved method above.

\section{A. Modification of Non-isothermal Simulation of RTM Process}

The case is provided by reference [19]. In the case, the part and position of resin inlet are shown in Fig 3.The improved calculation results are compared with the previous calculation results as shown in Figure 4.

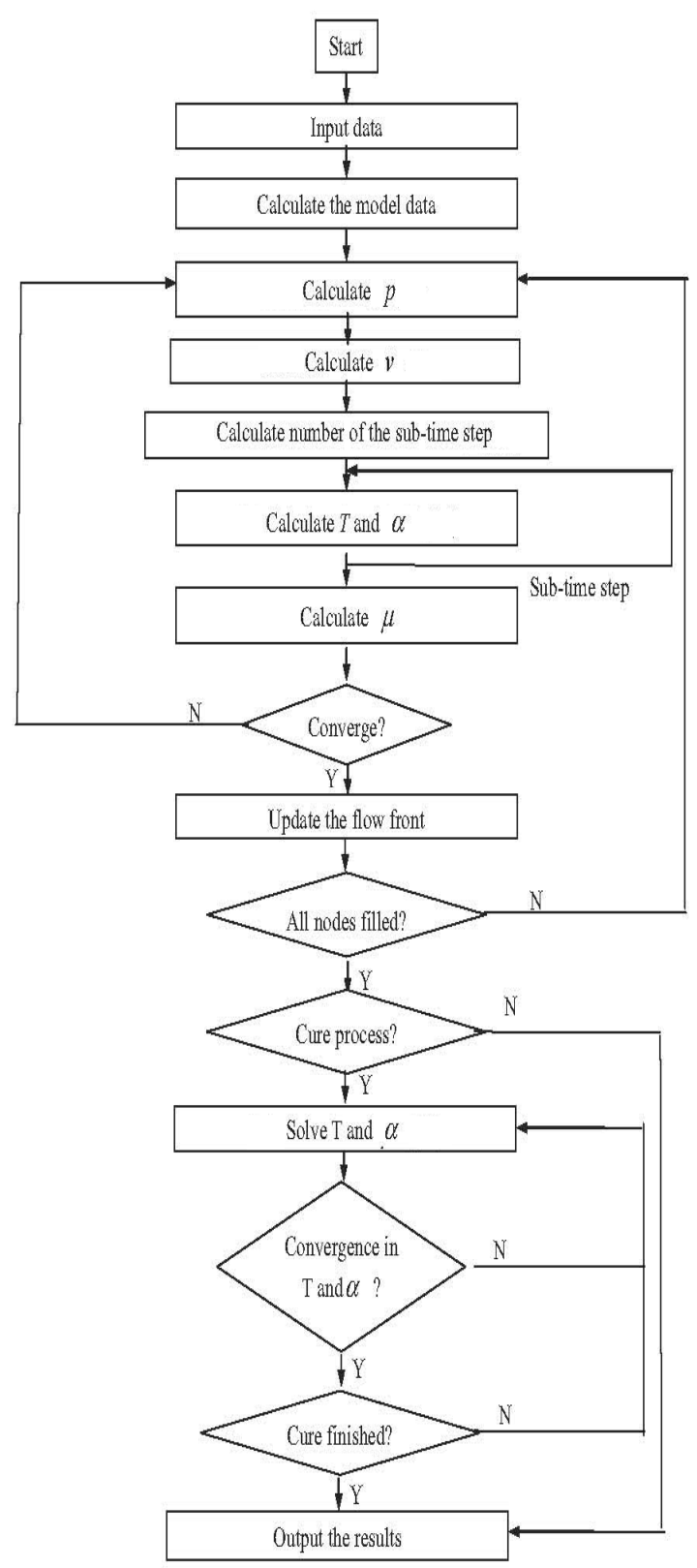

FIGURE II. THE FLOW CHART 
TABLE I. RESIN AND FIBER PARAMETERS ${ }^{[19]}$

\begin{tabular}{cc}
\hline parameters & Value \\
\hline$\rho_{\mathrm{f}} /\left(\mathrm{kg} \cdot \mathrm{m}^{-3}\right)$ & 2560 \\
$\rho_{\mathrm{r}} /\left(\mathrm{kg} \cdot \mathrm{m}^{-3}\right)$ & 1100 \\
$k_{\mathrm{f}} /\left(\mathrm{W} \cdot(\mathrm{m} \cdot \mathrm{K})^{-1}\right)$ & 0.0335 \\
$k_{\mathrm{r}} /\left(\mathrm{W} \cdot(\mathrm{m} \cdot \mathrm{K})^{-1}\right)$ & 0.168 \\
$c_{\mathrm{\rho f}} /\left(\mathrm{J} \cdot(\mathrm{kg} \cdot \mathrm{K})^{-1}\right)$ & 670 \\
$c_{\rho \mathrm{r}} /\left(\mathrm{J} \cdot(\mathrm{kg} \cdot \mathrm{K})^{-1}\right)$ & 1680 \\
$k_{\mathrm{x}}=k_{\mathrm{y}}=k_{\mathrm{z}} / \mathrm{m}^{2}$ & $2 \times 10^{-9}$ \\
$\phi$ & 0.7 \\
$\Delta H /\left(\mathrm{J} \cdot \mathrm{m}^{-3}\right)$ & $1.54 \times 10^{8}$ \\
$m_{1}$ & 0.3 \\
$\mu_{0} /\left(\mathrm{Pa}^{2} \mathrm{~s}\right)$ & $2.78 \times 10^{-4}$ \\
$\left.E_{\mu} /(\mathrm{J} \cdot \mathrm{mol})^{-1}\right)$ & 18000 \\
$\alpha$ & 0.1 \\
$\alpha_{\mathrm{g}}$ & 1.5 \\
$\mathrm{~d}_{2}$ & 1.0 \\
$A_{1} /\left(\mathrm{s}^{-1}\right)$ & $3.7833 \times 10^{5}$ \\
$A_{2} /\left(\mathrm{s}^{-1}\right)$ & $6.7833 \times 10^{5}$ \\
$E_{1} /\left(\mathrm{J} \cdot \mathrm{mol}^{-1}\right)$ & 54418 \\
$E_{2} /\left(\mathrm{J} \cdot \mathrm{mol} l^{-1}\right)$ & 50232 \\
$m_{2}$ & 1.7 \\
\hline & \\
\hline &
\end{tabular}

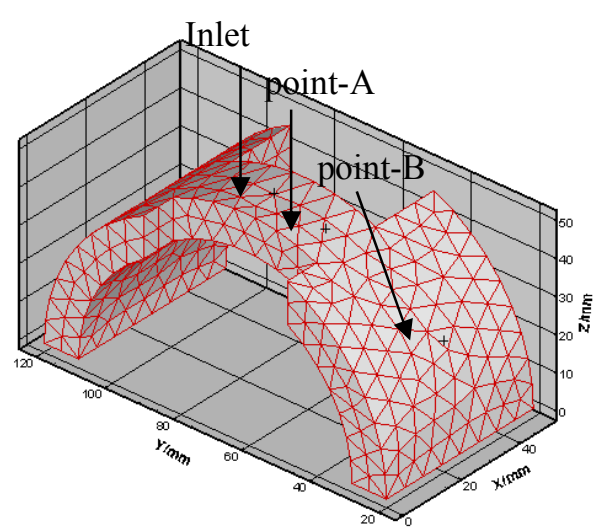

FIGURE III. THE PART AND POSITION OF RESIN INLET

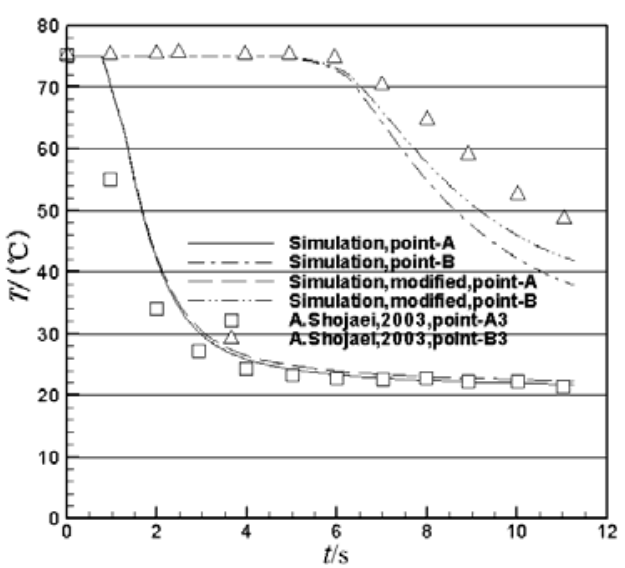

FIGURE IV. TEMPERATURE HISTORY CURVE OF TWO POINTS IN FILLING STAGE

Fig 4 for the corresponding temperature history of different points. Considering the cross diffusion in the common interface, the accuracy of the temperature field compared with previous work ${ }^{[15]}$ is effectively improved.

Fig 5 is the predicted temperature and cure degree of part at the end of filling. Based on the predicted data, This process can be better described and understood.

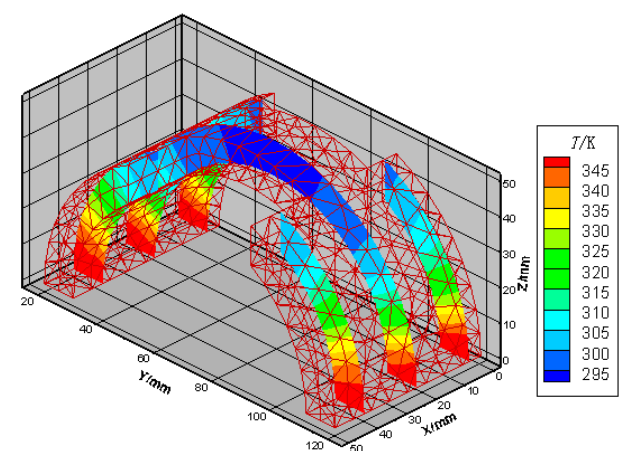

a: The temperature of part

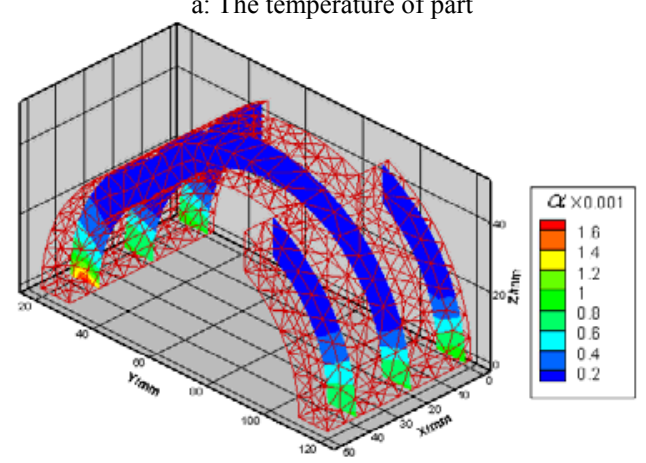

b: The cure degree of part

FIGURE V. THE PREDICTED TEMPERATURE AND CURE DEGREE OF PART AT THE END OF FILLING.

\section{B. Modification of Curing Process Simulation}

Another case is provided by reference [20]. The material parameters are described using table 2 and table 3 . The part is a flat with $15.24 \times 15.24 \times 25.4 \mathrm{~mm}$. A comparison between 
pervious works and current study was made with the temperature at the center of the plate, as Fig 6 shown.

TABLE II. MATERIAL PARAMETERS ${ }^{[20]}$

\begin{tabular}{ccc}
\hline & Resin & Fiber \\
\hline$\rho /\left(\mathrm{kg} \cdot \mathrm{m}^{-3}\right)$ & 1890 & 2540 \\
$c_{\rho} /\left(\mathrm{J} \cdot(\mathrm{kg} \cdot \mathrm{K})^{-1}\right)$ & 1260 & 840 \\
$k_{\mathrm{z}} /\left(\mathrm{W} \cdot(\mathrm{m} \cdot \mathrm{K})^{-1}\right)$ & 0.2163 & 0.01 \\
$k_{\mathrm{x}}, k_{\mathrm{y}} /\left(\mathrm{W} \cdot(\mathrm{m} \cdot \mathrm{K})^{-1}\right)$ & 0.4326 & $0.1,0.01$ \\
$\Delta H /\left(\mathrm{J} \cdot \mathrm{kg}^{-1}\right)$ & 77500 & \\
\hline $\mathrm{TABLE} \mathrm{III.} \mathrm{CURING} \mathrm{KINETIC} \mathrm{PARAMETERS}$ & \\
\hline $\mathrm{parameters}$ & Value \\
$A_{1} / \mathrm{s}^{-1}$ & 0 \\
$A_{2} / \mathrm{s}^{-1}$ & \\
$E_{1} /\left(\mathrm{J} \cdot \mathrm{mol}^{-1}\right)$ & $6.1667 \mathrm{X} 10^{20}$ \\
$E_{2} /\left(\mathrm{J} \cdot \mathrm{mol}^{-1}\right)$ & 0 \\
$M$ & $1.674 \mathrm{X} 10^{5}$ \\
$N$ & 0.524 \\
\hline
\end{tabular}

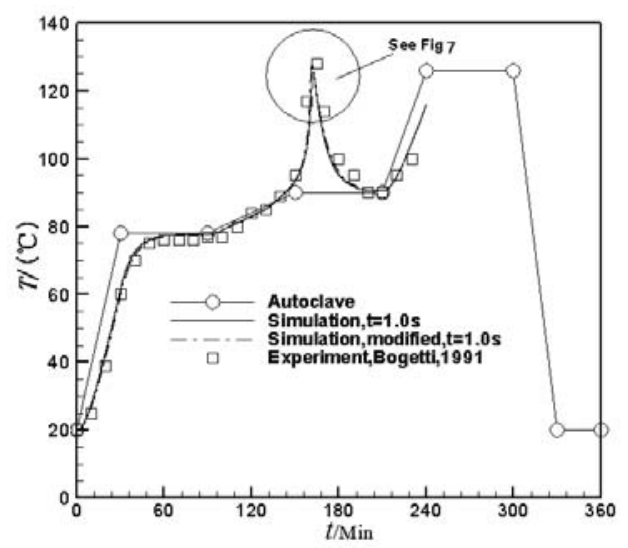

FIGURE VI. TEMPERATURE HISTORY CURVE OF THE CENTER POINT OF THE FLAT PLATE

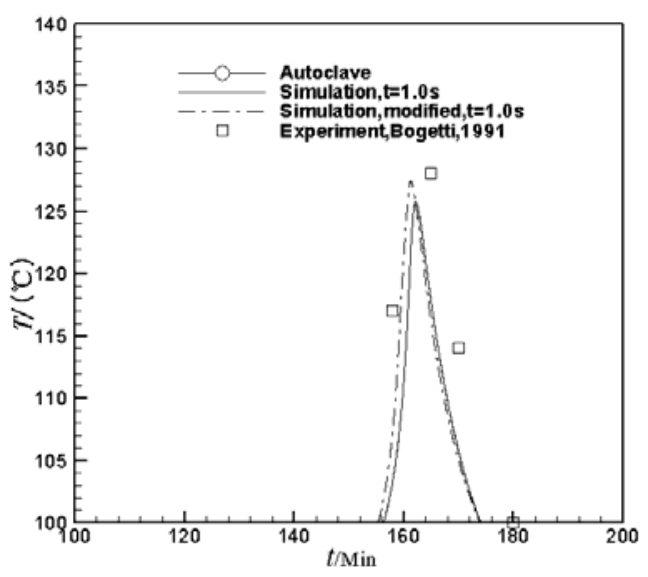

FIGURE VII. PARTIAL DETAIL FIGURE OF FIG 6
Considering revision, the predicted temperature peak is very close to experimental value, about $1{ }^{\circ} \mathrm{C}$ error which is reduced by approximately $2{ }^{\circ} \mathrm{C}$ than the original prediction error, as shown in Figure 6. Therefore, it can be said that considering the cross diffusion item in the discrete equation, the calculation precision is improved under not changing the calculation conditions.

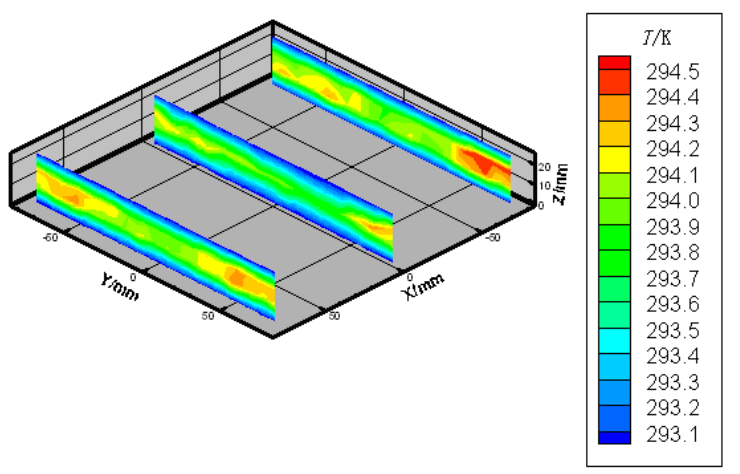

FIGURE VIII. THE TEMPERATURE AT THE END OF CURING

Fig. 8 accurately reflects the temperature inside and outside the part. Based on the temperature distribution, Product quality can be predicted accurately.

\section{CONCLUSION}

During the derivation of the diffusion term of the energy equation, the cross diffusion term appears on the common interface between control volumes when they are not orthogonal, but the calculation is usually ignored in the past. In this paper, an approximate calculation formula of the cross diffusion term between non-orthogonal control volumes is proposed in order to improve the accuracy of numerical calculation. In the end, two numerical examples show that the method can effectively improve the accuracy of numerical calculation of temperature field.

\section{ACKNOWLEDGEMENTS}

This work was supported by Educational Commission of Zhejiang Province of China ((program no: Y201533757), Natural Science Foundation of Ningbo City (program no: 2015A610066) and State Key Laboratory of Materials Processing and Die \& Mould Technology(P2017-009), Huazhong University of Science and Technology.

\section{REFERENCE}

[1] A.W. Chan, R.J. Morgen, Computer modeling of liquid composite molding for 3-dimesional complex shaped structures, in: Proceedings of the 10th ASM/ESD Advanced composites Conference, Dearborn, USA, 1994, pp. 341-345.

[2] M.K. Kang, W.I. Lee, S.M. Cho, Simulation of mold filling process during resin transfer molding, Journal of Materials Process and manufacturing Science 3 (1995) 291-313.

[3] M.V. Bruschke, S.G. Adani, A numerical approach to model non-isothermal viscous flow through fibrous media with free surfaces, International Journal of Numerical Method in Fluids 19 (1994) 575-603.

[4] R.J. Lin, L.J. Lee, M.L. Liou, Non-isothermal mold filling and curing simulation in thin cavities with prep laced fiber mats, International Polymer Process 6 (4)(1991) 356-369. 
[5] L.J. Lee, W.B. Young, R.J. Lin, Mold filling and curing modeling of RTM and SRIM processes, Composite Structure 27 (1994) 109-120.

[6] F. Trochu, R. Gauvin, D.M. Gao, Numerical analysis of the resin transfer molding process by the finite element method, Advances in Polymer Technology 12 (4) (1993) 329-342.

[7] Ren-liang Chen, Bing Gui, Ming-cheng Li, Zhi-yong Liang, Non-isothermal mold filling and curing simulation for resin transfer molding, Chinese Journal of the Aeronautics 16 (4) (2003) 247-252.

[8] D.M. Gao, F. Trochu, R. Gauvin, Heat transfer analysis of non-isothermal resin transfer molding by the finite element method, Materials and manufacturing process 10 (1995) 57-64.

[9] Y.C. Lam, S.C. Joshi, X.L. Liu, Numerical simulation of the mold filling process in resin-transfer molding, Composite Science and Technology 60 (2000) 845-855.

[10] Seong Taek Lim, Woo Il Lee, An analysis of the three-dimensional resin transfer mold filling process, Composites Science and Technology 60 (2000) 961-975.

[11] A. Shojaei, S.R. Ghaffarian, S.M.H. Karimian, Three-dimensional process cycle simulation of composite parts manufactured by resin transfer molding, Composite Structures 65 (2004) 381-390.

[12] A. Cheung, Y. Yu, K. Pochiraju, Three-dimensional finite element simulation of curing of polymer composites, Finite Elements in Analysis and Design 40 (2004) 895-912.

[13] S.C. Joshi, Y.C. Lam, X.L. Liu, Mass conservation in numerical simulation of resin flow, Composites Part A: Applied Science and Manufacturing 31 (2000) 1061-1068.

[14] Chow P, Cross M, Pericelous K. A natural extension of the conventional finite voulume method into polygonal unstructured meshes for CFD application [J]. App.Math.Modeling, 1996, 20:170-183.

[15] Fei Shi, Xianghuai Dong, 3D numerical simulation of filling and curing processes in non-isothermal RTM process cycle[J], Finite Elements in Analysis and Design, 2011,47:764-770.

[16] Fei Shi, Xianghuai Dong, Numerical study of resin transfer molding (RTM) curing process[J], Front. Mater. Sci. China, 2010, 4(2): 217-224

[17] Fei Shi, Xianghuai Dong, Numerical simulation of non-isothermal RTM process[J], Acta Materiae Compositae Sinica, 2009,26(4):146-150.

[18] Fei Shi, Xianghuai Dong, Numerical simulation of curing procedure of three-dimensional RTM $[\mathrm{J}]$, Acta Materiae Compositae Sinica, 2010,27(2):90-94.

[19] Shojaei A, Ghaffarian S R, Karimian S M H. Simulation of the three-dimensional non-isothermal mold filling process in resin transfer molding [J]. Composites Science and Technology, 2003, 63(13):1931-1948.

[20] Bogetti T A, Gillespie Jr J W. Two- dimensional cure simulation of thick thermosetting composites [J]. Journal of Composite Materials, 1991, 25 (3):239 - 273. 